\title{
Importancia de los electrolitos y la hidratación en la actividad física
}

\author{
Importance of electrolytes and hydration in physical activity
}

Araceli Ortiz-Polo ${ }^{a}$, Mónica S. Carrasco-García ${ }^{b}$, Lisett Hernández-Ponce ${ }^{c}$

\begin{abstract}
:
Introduction: A good hydration is essential for health because influences the correct function of the body of athletes. In all sports practice there is a decrease hydroelectrolytic by the loss of water in the form of sweat and by the increase in breathing, which could affect performance, endurance, strength and muscle power among other important damage derived from the state of dehydration. Objective: describe the importance of electrolytes and hydration in physical activity, through a bibliographic analysis, to understand their relevance in the physiological state of the athlete. Methodology: A bibliographic review was conducted in specialized search engines such as Google Academic, Scielo, Redalyc, Pub Med, Elservier, Academia.edu and Dialnet, in which articles, books and web pages were analyzed. Results: The main electrolytes that play an important role in physical activity are essentially. Sodium: Excessive sweating during exercise can reduce sodium and chlorine levels by 5-7\%. Failure to replenish itself can result in a state of hyponatremia, leading to general weakness, muscle cramps, headache and nausea. Potassium: Excessive sweating can reduce levels by $1 \%$, causing muscle weakness or heart condition and in extreme cases irreversible cardiovascular, muscle and kidney injuries. Conclusion: a good hydration should be taken care of before, during and after sports, followed by an adequate electrolyte charge mainly of sodium and potassium ions, which perform multiple physiological functions in muscle activity, and without which can lead to a decrease in athletic performance, anatomical injuries and the general health of the athlete.
\end{abstract}

Keywords:

Hydration, Electrolytes, Sodium, Potassium, Physical Activity, Athletes, Performance.

\section{Resumen:}

Introducción: El buen estado de hidratación es fundamental para la salud ya que influye en la correcta función del organismo de los deportistas. En toda práctica deportiva se produce una disminución hidroelectrolítica por la pérdida de agua en forma de sudor y por el incremento en la respiración, pudiendo afectar el rendimiento, la resistencia, la fuerza y potencia muscular entre otros daños importantes derivados del estado de deshidratación. Objetivo: Describir la importancia de los electrolitos y de la hidratación en la actividad física, mediante un análisis bibliográfico, para comprender su relevancia en el estado fisiológico del deportista. Metodología: Se realizó una revisión bibliográfica en los buscadores especializados como Google Académico, Scielo, Redalyc, Pub Med, Elservier, Academia.edu y Dialnet, en el que se analizaron artículos, libros y páginas web. Resultados: Los electrolitos principales que juegan un papel importante en la actividad física se encuentran esencialmente el Sodio: Una excesiva sudoración durante el ejercicio puede reducir los niveles de sodio y cloro en un 5-7\%. Si no se repone se puede producir un estado de hiponatremia, y dar lugar a debilidad general, calambres musculares, dolor de cabeza y náuseas. Potasio: Una excesiva sudoración puede reducir los niveles un $1 \%$, provocando debilidad muscular o afecciones cardiacas y en casos extremos lesiones cardiovasculares, musculares y renales de tipo irreversible. Conclusión: Una correcta hidratación se debe cuidar antes, durante y después de la práctica deportiva, seguida de una carga electrolítica adecuada sobre todo de iones sodio y potasio, los cuales cumplen múltiples funciones fisiológicas en la actividad muscular, y sin los cuales se puede ocasionar una disminución en el rendimiento deportivo, lesiones anatómicas y afecciones en el estado de salud general del deportista.

Palabras Clave:

Hidratación, Electrolitos, Sodio, Potasio, Actividad Física, Deportistas, Rendimiento.

\footnotetext{
${ }^{a}$ Autor de correspondencia, Universidad Autónoma del Estado de Hidalgo, Instituto de Ciencias de la Salud, ORCID: 0000-0001-55612221,Email: araceli_ortiz4208@uaeh.edu.mx

${ }^{\mathrm{b}}$ Universidad Autónoma del Estado de Hidalgo, Instituto de Ciencias de la Salud, Email: m.suheily11@gmail.com

${ }^{\text {b } U n i v e r s i d a d ~ A u t o ́ n o m a ~ d e l ~ E s t a d o ~ d e ~ H i d a l g o, ~ I n s t i t u t o ~ d e ~ C i e n c i a s ~ d e ~ l a ~ S a l u d, ~ E m a i l: ~ l i z h p 97 @ g m a i l . c o m ~}$
} 


\section{Introducción}

La práctica deportiva, tanto profesional, como de principiantes, ha alcanzado mayor auge en las últimas décadas. El incremento de sitios para realizar actividades físicas y deportivas son más notorios en el desarrollo de las sociedades, ya sean espacios públicos o privados. Hoy en día, un sin número de personas realizan ejercicio sin orientación de un profesional de las ciencias del deporte, exponiéndose a sufrir alteraciones como deshidratación y pérdida electrolítica por desconocimiento de la realización correcta del ejercicio físico.

Las competiciones deportivas cada vez más tienen una mayor exigencia en cuanto a la intensidad del esfuerzo, precisando controlar minuciosamente todos los aspectos que afectan al rendimiento deportivo (Urdampilleta, López-Grueso, Martínez-Sanz y Mielgo-Ayuso, 2014).

Es fundamental mantener un equilibrio entre la ingesta y la pérdida de agua corporal, es decir, un estado óptimo de hidratación, en los deportistas, antes, durante y después de la práctica deportiva.

La euhidratación es el estado adecuado de hidratación y es importante para mantener la salud y promover el rendimiento durante la actividad física. Pérdidas de agua corporal, incluso en pequeñas cantidades, pueden tener un efecto negativo en el organismo, afectando en los deportistas la fuerza muscular, la resistencia y el consumo máximo de oxígeno (Mielgo-Ayuso et al., 2015), por lo tanto, la deshidratación, puede tener consecuencias nocivas en la salud, tanto en deportista de élite, así como novatos.

Cuando se realiza actividad física, se presentan modificaciones del equilibrio hidroelectrolítico, fundamentalmente a causa de la pérdida de agua por el sudor y la respiración (Urdampilleta et al., 2014). El incremento en la tasa de sudoración al realizar actividad física como resultado de la regulación de la temperatura corporal y el aumento de la frecuencia respiratoria provoca esta gran pérdida de agua corporal.

También pueden producirse pérdidas considerables de líquidos y electrolitos durante la práctica de ejercicio en periodos prolongados de tiempo y en condiciones ambientales y climáticas de alta temperatura y concentraciones de humedad relativa bajas. En estas circunstancias, la ingesta inadecuada de líquidos durante la práctica del ejercicio de resistencia puede conducir a la deshidratación e hiponatremia (Cepero, Padial, Rojas, Romero y De la Cruz, 2016).
La tasa de sudoración (TS), es la medida de la pérdida de agua durante la actividad física, depende de factores como las condiciones del medio ambiente (temperatura y humedad), la genética y el acondicionamiento del deportista.

El sudor está compuesto por agua (99\%), electrolitos y compuestos nitrogenados. Una excesiva sudoración durante el ejercicio puede reducir los niveles de sodio y cloro en un 5-7\%, y de potasio un $1 \%$, por ello deben ser repuestos con el fin de evitar una depleción y con ellos una deshidratación hidroelectrolítica (Mielgo-Ayuso et al., 2015). Es decir, que para evitar la pérdida excesiva de agua y de electrolitos mientras se practica actividad física, se deben recuperar éstos químicos mediante la ingesta de líquidos.

Como señalan Mielgo-Ayuso et al. (2015) la reposición de agua y de electrolitos debe realizarse, primera y principalmente, a través de agua o en ciertos casos por medio de bebidas deportivas u otras bebidas específicas, con el fin de evitar la deshidratación, la cual puede tener un efecto negativo en el sistema cardiovascular y termorregulador, además de comprometer a los sistemas metabólico, endocrino y excretor.

Por tanto, el objetivo de este análisis es describir la importancia de los electrolitos y de la hidratación en la actividad física, mediante un análisis bibliográfico, para comprender su relevancia en el estado fisiológico del deportista.

\section{Metodología}

Se realizó una revisión bibliográfica en los buscadores especializados Google Académico, Scielo, Redalyc, Pub Med, Elservier, Academia.edu y Dialnet, así como en revistas especializadas en temas deportivos y Medicina Deportiva, entre los que se revisaron artículos, libros y páginas web. La revisión bibliográfica se realizó en lengua hispana e inglesa.

Se incluyeron todas las fuentes bibliográficas que contenían las palabras clave y se excluyeron aquellos que se publicaron antes del año 2014.

\section{Resultados}

El contenido de agua corporal es de aproximadamente el $60 \%$ del peso corporal, pero varía a lo largo de la vida en función de distintos factores como la edad, el género, composición muscular, entre otros. 
El agua corporal se localiza, mayoritariamente, en dos grandes compartimentos: como agua intracelular (donde se encuentran $2 / 3$ partes del total), y como agua extracelular (que contiene $1 / 3$ parte de total), repartida entre el espacio intersticial, la sangre, la linfa, las secreciones etc. Los varones, sobre todo si son deportistas, poseen un mayor componente muscular y por consiguiente un mayor contenido en agua, dado que el músculo es un tejido comparativamente mucho más rico en agua que el tejido adiposo (70\% versus 10\%) (Lizarraga, 2015).

Para la evaluación del estado de hidratación en el deporte y el ejercicio existen diferentes marcadores biológicos, los más usados son: masa corporal (medida de diferencia entre el peso del deportista antes y después del ejercicio), osmolaridad específica de la orina, gravedad específica de la orina y la osmolaridad plasmática (Castro-Sepúlveda et al., 2015).

Por lo anterior, en el deporte de alto rendimiento, se hace imprescindible un estudio minucioso sobre la influencia del ejercicio en las adaptaciones fisiológicas, especialmente en el estado de hidratación y la TS.

\section{Fisiología de la TS}

Durante la práctica de ejercicio físico, dependiendo de la intensidad del trabajo a realizar, de las condiciones ambientales (temperatura y humedad) y del tipo de ropa utilizada (materiales y tecnología), puede ascender la carga térmica y producirse un importante aumento en la temperatura corporal; por lo cual, como mecanismo de control, se produce un incremento en el flujo sanguíneo hacia la piel y, consecuentemente, una elevación en la producción del sudor.

La evaporación del sudor es la vía primaria de pérdida de calor que revierte en una continua pérdida de líquidos corporales y electrolitos, principalmente sodio y potasio (Marins et al., 2018).

Las pérdidas de fluidos durante el ejercicio y el deporte pueden llegar hasta del $5 \%$ del peso corporal, lo cual puede perjudicar la calidad del entrenamiento, o el rendimiento en la competición e incluso la salud (Salazar y Barboza, 2016).

Nebot et al. (2015) aseguran que la pérdida de fluidos por la sudoración tiende a limitar el flujo sanguíneo al músculo a través de una reducción del volumen plasmático y el volumen sistólico en cada latido, por lo que el mismo autor, reporta que existen evidencias de una disminución del rendimiento deportivo con tan sólo pérdidas del $1 \%$ del peso corporal por un aumento del trabajo cardiaco, y pérdidas de peso corporal superiores al $2 \%$ afectan sobre todo al ejercicio aeróbico en climas cálidos, además de disminuir el rendimiento mental y cognitivo.

La humedad relativa y el calor, ocasionan patologías musculares y sistémicas por pérdidas de fluidos. El rendimiento de los deportistas podría disminuir si no se considera que un $80 \%$ de la energía empleada en la contracción muscular es liberada en forma de calor y que el reflejo de la sed es una respuesta ante la deshidratación.

Es importante señalar que no solo se pierde agua en el sudor, sino que también sales minerales esenciales como son los electrolitos sodio $(\mathrm{Na}+)$ y potasio $(\mathrm{K}+)$. La disminución de estos, puede inducir a una hiponatremia o hipokalemia, y afectar el rendimiento del deportista, así lo señalan Castro-Sepúlveda, Astudillo, Mackay y Jorquera (2016), quienes refieren que también se generan efectos negativos a su salud general del deportista.

Salazar y Barboza (2016) mencionan que la deshidratación progresiva durante el ejercicio es frecuente puesto que muchos deportistas no ingieren suficientes fluidos para reponer las pérdidas producidas en esta actividad. Esto no sólo va a provocar una disminución del rendimiento físico, sino que además aumenta el riesgo de lesiones, y puede poner en juego la salud e incluso la vida del deportista.

El rendimiento en el deporte depende de un gran número de factores, tales como técnicos, tácticos, físicos, fisiológicos y psicológicos, aunque es importante considerar que estos pueden verse afectados por la deshidratación. Castro-Sepúlveda et al. (2015) indican además, que los estados de hidratación deficientes, no solo afectan el rendimiento deportivo, sino que también pueden afectar la salud del deportista, induciendo a alteraciones desde leves como edemas, salpullido, calambres, entre otras, hasta potencialmente mortales como un golpe de calor.

Para Vega-Pérez, Ruiz-Hurtado, Macías-González, García-Peña, y Torres-Bugarín (2016) la hidratación del atleta va a depender de las estrategias hídricas utilizadas antes, durante y después del ejercicio. La euhidratación es condición fundamental para optimizar el rendimiento deportivo. La importancia de los líquidos, el agua y las bebidas para deportistas (bebidas isotónicas y bebidas de recuperación) radica en el restablecimiento de la homeostasis del organismo por la pérdida de agua y 
electrolitos por la actividad física a través de mecanismos como la sudoración.

\section{Electrolitos}

\section{Sodio $\left(\mathrm{Na}^{+}\right)$}

La Asociación Mexicana de Educación Deportiva (s.f a) define al sodio como el principal catión del medio extracelular. Asociado al cloro y a los bicarbonatos, tiene gran importancia en el equilibrio acidobásico. Su misión esencial es mantener la presión osmótica en el medio extracelular y evitar así una pérdida excesiva de agua. EI sodio también tiene cierta importancia en el mantenimiento de la excitabilidad normal del músculo y en la permeabilidad del aporte.

El sodio es un nutriente esencial, imprescindible para la vida, que permite al organismo mantener el equilibrio iónico y retener agua para conseguir una buena hidratación. Por este motivo resulta primordial para la persona que realiza esfuerzos intensos o duraderos, y en cualquier otra situación en la que se suda de manera abundante, porque hace mucho calor y/o el grado de humedad ambiental es alto (Palacios, 2014). Una excesiva sudoración durante el ejercicio puede reducir los niveles de sodio y cloro en un 5-7\% (Mielgo-Ayuso et al., 2015).

El exceso de sodio es causa de retención de agua, mientras que su déficit provoca una pérdida de la misma (AMED, s.f b). Los rangos de normalidad de sodio, pueden verse afectados por las causas que se observan en la tabla 1.

Tabla 1: Parámetros de sodio y su significado fisiopatológico

\begin{tabular}{|l|l|l|l|}
\hline Parámetro & $\begin{array}{l}\text { Valor normal } \\
(\mathrm{mEq} / \mathrm{l})\end{array}$ & Elevación & Disminución \\
\hline Sodio & $\begin{array}{l}\text { En orina: } \\
40-220\end{array}$ & Deshidratación & $\begin{array}{l}\text { Hipersudora- } \\
\text { ción }\end{array}$ \\
\cline { 2 - 4 } & $\begin{array}{l}\text { En sangre: } \\
135-145\end{array}$ & Deshidratación & $\begin{array}{l}\text { Vómito } \\
\text { Diarrea }\end{array}$ \\
\hline
\end{tabular}

Nota: Valores normales de sodio y las causas de su elevación y disminución en el organismo. Adaptado de «La nutrición en la actividad física y el deporte», de Sirvent y Alvero, 2017, p. 244251. Alicante, España: (C) Universidad de Alicante.

Un deportista de resistencia entrenado, en competición, puede llegar a perder más de dos litros de sudor en una hora.
Este sudor contiene, además de agua, una cantidad importante de sodio (entre 20 y 50 milimoles /litro o 400 a $1.000 \mathrm{mg}$ de por litro de sudor aproximadamente, según las características de la persona) (Palacios, 2014).

No reponer este mineral, se puede producir hiponatremia, y dar lugar a síntomas como debilidad, calambres musculares, dolor de cabeza, náuseas; es decir, cuando exista sudoración excesiva, conviene restaurar lo antes posible el agua y el sodio eliminados, para evitar pérdida de rendimiento físico y psíquico y un estado de deshidratación.

Campbell (2014), menciona que la recomendación adecuada de sodio es de 1500 mg / día, estos rangos dependen del deportista, su tiempo de entrenamiento y el ambiente, ya que si pierde más sodio mediante la sudoración, se necesitará mayor cantidad de lo recomendado para evitar calambres musculares y en casos más extremos posibles estados de hiponatremia en el deportista.

\section{Potasio $\left(\mathrm{K}^{+}\right)$}

Así como el sodio es el principal catión del medio extracelular, el potasio lo es del medio intracelular. Sus principales funciones son la regulación del contenido de agua en la célula, activación de los sistemas enzimáticos y aumento de la excitabilidad neuro-muscular. La glucogenólisis se acompaña de la liberación de potasio, mientras que la formación del glucógeno provoca un almacenamiento del mismo. Cuando existe catabolismo proteico, el potasio sale de las células. En caso de anabolismo, se produce la situación inversa (AMED, s.f b).

En la tabla 2 se muestran las causas de alteración del parámetro normal de potasio en sangre.

Tabla 2: Parámetros de potasio y su significado fisiopatológico

\begin{tabular}{|l|l|l|l|}
\hline Parámetro & $\begin{array}{l}\text { Valor normal } \\
(\mathrm{mEq} / \mathrm{l})\end{array}$ & Elevación & Disminución \\
\hline Potasio & $\begin{array}{l}\text { En sangre: } \\
3.7-5.2\end{array}$ & $\begin{array}{l}\text { Deshidrata- } \\
\text { ción } \\
\text { Suplementos } \\
\text { de potasio }\end{array}$ & $\begin{array}{l}\text { Ayuno } \\
\text { prolongado } \\
\text { Vómito } \\
\text { Diarrea }\end{array}$ \\
\hline
\end{tabular}

Nota: Valores normales de potasio y las causas de su elevación y disminución en el organismo. Adaptado de «La nutrición en la actividad física y el deporte», de Sirvent y Alvero, 2017, p. 244. Alicante, España: (C)Universidad de Alicante. 


\section{Hidalgo, Vol. 8, No. 15 (2019) 241-246}

Una excesiva sudoración durante el ejercicio puede reducir los niveles de potasio un 1\% (Mielgo-Ayuso et al., 2015). La pérdida de potasio puede provocar debilidad muscular o afecciones cardiacas. En casos de déficit extremo, se pueden producir lesiones cardiovasculares, musculares y renales de tipo irreversible (Urdampilleta et al., 2014).

En el artículo de Pacheco, González y Romeu (2018) se especifica que, según la OMS, la ingesta diaria de potasio en adultos sanos no debería ser inferior a $3.510 \mathrm{mg}$ de potasio/día en un adulto activo, por lo tanto, esta dosis mínima también deberá mantenerse en un deportista.

\section{Rehidratación}

\section{Bebidas rehidratantes}

La asociación de hidratos de carbono $(\mathrm{HC})$ y sodio en el agua facilita el transporte de esta al interior de las células intestinales, lo que ayuda a conseguir una hidratación más rápida y adecuada, por lo que la ingesta de bebidas con una mezcla de electrolitos y carbohidratos ayudan a reponer la pérdida de líquidos y electrolitos producida durante la sudoración en la actividad física, previenen la reducción del volumen plasmático, regulan la sensación de sed y retrasan la aparición de fatiga, con el consiguiente beneficio en el rendimiento (Nebot, 2015).

Sirvent y Alvero (2017), expresan que después de ejercicios de larga duración y/o intensidad es recomendable una bebida con sodio para aumentar la retención de líquido y la mejor forma de administrar el agua $y$ el sodio perdido es mediante una bebida adecuada, que debe contener entre $46-115 \mathrm{mg}$ de sodio por $100 \mathrm{~mL}$.

La osmolaridad ( $\mathrm{mOsm} / \mathrm{kg}$ ), se entiende como la medida que expresa el nivel de concentración de los componentes de diversas disoluciones. Su clasificación, según la Universidad Internacional de La Rioja (2018), indica que las bebidas que tengan una osmolaridad cercana a 300 miliosmoles se denominan isotónicas, las que tengan por debajo hipotónicas y las que están por encima hipertónicas.

Palacios (2014) señala que para acelerar la hidratación de las células se recomienda que esta bebida aporte hidratos de carbono de alto índice glucémico (1,5-8,75 g/100 ml) y cuya osmolaridad, sea hipotónica (200-270 $\mathrm{mOsm} / \mathrm{kg}$ agua) o isotónica (270-330 mOsm/kg agua). El autor sugiere que todas las bebidas necesitan transformarse en isotónicas para poder ser absorbidas. De ahí que resulte importante una osmolalidad adecuada, pues esta transformación precisa de un tiempo, el cual es crucial si se quiere mantener la hidratación y el rendimiento deportivo, lo que no se consigue cuando se utilizan bebidas hipertónicas (>330 $\mathrm{mOsm} / \mathrm{kg}$ agua), dado que retrasarán el vaciado gástrico y por tanto la absorción rápida de carbohidratos y resto de sustancias, así como las marcadamente hipotónicas (<200 mOsm/kg agua).

\section{Conclusión}

Con el propósito de alcanzar las metas planteadas, los deportistas deben optar por técnicas y programas que les ayuden a cubrir las necesidades que la actividad física exige, entre los que se encuentra una adecuada nutrición, dentro de la cual, la hidratación juega un papel trascendental para mantener la homeostasis y con ello un mejor rendimiento deportivo, por lo que vigilar el estado de hidratación antes, durante y después de la práctica física, es indispensable en todo deportista. Por otra parte, se pretende evitar la pérdida excesiva de agua y de electrolitos, como sodio y potasio, o bien, buscar una recuperación óptima, a través de bebidas rehidratantes, previniendo la disminución del rendimiento físico, lesiones musculares e incluso afecciones neurológicas en la salud del individuo. Por lo tanto, es preciso que el deportista cuente con un equipo multidisciplinario, en el que el rol de un nutriólogo, será imprescindible para cumplir los objetivos establecidos.

\section{Referencias}

[1] Asociación Mexicana de Educación Deportiva. (s.f. a). Curso principios nutrición aplicada al culturismo y deporte. Recuperado de https://amedweb.com/recursos/PN.pdf

[2] Asociación Mexicana de Educación Deportiva. (s.f. b). Nutrición Aplicada al Culturismo y Deporte. Recuperado de

https://s3.amazonaws.com/academia.edu.documents/50716086/PRINC IPIOS_NUTRICION_APLICADA_AL_CULTURISMO.pdf?response -content-

disposition=inline\%3B\%20filename\%3DNutricion_aplicada_al_cultur ismo_y_depor.pdf\&X-Amz-Algorithm=AWS4-HMAC-SHA256\&XAmz-

Credential=AKIAIWOWYYGZ2Y53UL3A\%2F20190821\%2Fuseast-1\%2Fs3\%2Faws4_request\&X-Amz-

Date $=20190821 \mathrm{~T} 192022 Z \& X-A m z-$ Expires $=3600 \& X-A m z-$ SignedHeaders $=$ host $\&$ X-Amz-

$\underline{\text { Signature }=7 \mathrm{~d} 30585 \mathrm{c} 5 \mathrm{~d} 6575 \mathrm{fab}} \mathrm{a} 48 \mathrm{~d} 2 \mathrm{e} 024 \mathrm{a} 78102 \mathrm{e} 6 \mathrm{af} 456 \mathrm{a} 20 \mathrm{a} 864 \mathrm{be} 8 \mathrm{~b}$ 5ecf7f4bad0a23

[3]Campbell, B. (2014). Sports Nutrition: Enhancing Athletic Performance. Taylor \& Francis Group. Recuperado de https://books.google.com.ec/books?lr=\&hl=es\&id=jULBAQAAQBAJ $\& \mathrm{q}=\mathrm{La}+\mathrm{recomendaci} \% \mathrm{C} 3 \% \mathrm{~B} 3 \mathrm{n}+\mathrm{adecuada}+\mathrm{de}+$ sodio+es+de+1500+m $\mathrm{g}+\% 2 \mathrm{~F}+\mathrm{d} \% \mathrm{C} 3 \% \mathrm{ADa} \% 2 \mathrm{C}+$ estos+rangos+dependen+del+deportista $\% 2$ $\mathrm{C}+\mathrm{su}+$ tiempo+de+entrenamiento+y+el+ambiente $\% 2 \mathrm{C}+\mathrm{ya}+\mathrm{que}+\mathrm{si}+\mathrm{pie}$ rde+m\%C3\%A1s+electrolitos\%2C+es+decir\%2C+m\%C3\%A1s+sodio + mediante+la+sudoraci\% $3 \% \mathrm{~B} 3 \mathrm{n} \% 2 \mathrm{C}+$ se + necesitar $\% \mathrm{C} 3 \% \mathrm{~A} 1+$ mayo

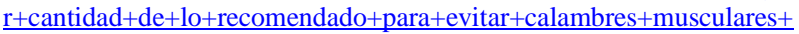
$\mathrm{y}+\mathrm{en}+\mathrm{casos}+\mathrm{m} \% \mathrm{C} 3 \% \mathrm{~A} 1 \mathrm{~s}+$ extremos+posibles+estados+de+hiponatre $\underline{\text { mia+en+el+deportista+\#v=onepage } \& \mathrm{q}=\text { =sodium } \% 20 \& \mathrm{f}=\text { false }}$ 
[4] Castro-Sepúlveda, M., Astudillo, S., Alvarez, C., Zapata-Lamana, R., Zbinden-Foncea, H., Ramírez-Campillo, R. y Jorquera, C. (2015). Prevalencia de deshidratación en futbolistas profesionales chilenos antes del entrenamiento. Nutrición Hospitalaria, 32(1), 308-311. doi: 10.3305/nh.2015.32.1.8881

[5] Castro-Sepúlveda, M., Astudillo, S., Mackay, K. y Jorquera, C. (2016). El consumo de leche posterior al ejercicio disminuye la excreción de electrolitos. Revista Internacional de Medicina y Ciencias de la Actividad Física y el Deporte, 16(62), 221-228. doi: http://dx.doi.org/10.15366/rimcafd2016.62.003

[6] Cepero, M., Padial, R. Rojas, F. J., Romero, D. y De la Cruz, J. C. (2016). Efectos de bebidas carbohidratadas y proteicas sobre la recuperación del esfuerzo. Revista Internacional de Medicina y Ciencias de la Actividad Física y el Deporte, 16(62), 373-401. Recuperado de Http://cdeporte.rediris.es/revista/revista62/artefectos701.htm

[7] Lizarraga, M. A. (2015). Hidratación en diversas edades y actividades deportivas. Revista de la Reial Acadèmia de Medicina de Catalunya, 30 (3), 126-129. Recuperado de https://www.raco.cat/index.php/RevistaRAMC/search/results?query=li zarraga\&searchField $=$

[8] Marins, J. C., Pereira, L., Amorim, P. R., Arnaiz-Lastras, J., SilleroQuintana, M. y Alfenas, C. R. (2016). Suplementos de carbohidratos durante un ejercicio: efectos sobre los electrolitos y glucosa. Revista Internacional de Medicina y Ciencias de la Actividad Física y el $\begin{array}{llll}\text { Deporte, } & \text { 18(70): } & \text { 269-287. }\end{array}$ http://dx.doi.org/10.15366/rimcafd2018.70.005

[9] Mielgo-Ayuso, J., Maroto-Sánchez, B., Luzardo-Socorro, R., Palacios, G., Palacios, N. y González-Gross, M. (2015). Valoración del estado nutricional y del gasto energético en deportistas. Revista Española de Nutrición Comunitaria, 21(Supl.1), 225-234. doi: 10.14642/RENC.2015.21.sup1.5069

[10] Nebot, V., Drehmer, E., Elvira, L., Sales, S., Sanchís, C., Esquius, L. y Pablos, A. (2015). Efectos de la ingesta voluntaria de líquidos (agua y bebida deportiva) en corredores por montaña amateurs. Nutrición Hospitalaria, 32(5): 2198-2207.doi:10.3305/nh.2015.32.5.9637

[11] Pacheco, R. P., González, M. y Romeu, M. (2018). Estudio Nutri-K: Evaluación de la ingesta de potasio y el deporte en adultos jóvenes. Nutrición clínica y dietética hospitalaria, 38(3), 161-167. doi: $10.12873 / 383$ romeu

[12] Palacios, N. (2014). El sodio de la sal: necesidades en el deportista Archivos de Medicina del Deporte, 31(6), 376-377. Recuperado de http://archivosdemedicinadeldeporte.com/articulos/upload/editorial 16 $\underline{4 . p d f}$

[13] Salazar, J. P. y Barboza, J. N. (2016). Valores de Sodio y Potasio en suero y su impacto en variación pre y pos ejercicio de actividades fuertes en deportistas. Revista de Ciencias Biológicas y de la Salud. 18 (2), 24-26. doi: http://dx.doi.org/10.18633/bt.v18i2.276

[14] Sirvent, J. y Alvero, J. (2017). La nutrición en la actividad física y el deporte. Recuperado de https://books.google.com.mx/books?id=Lxs DwAAQBAJ\&printsec=f rontcover\&dq=LA+NUTRICI\%C3\% $93 \mathrm{~N}+\mathrm{EN}+\mathrm{LA}+\mathrm{ACTIVIDAD}+\mathrm{F} \%$ C3\%8DSICA+Y+EL+DEPORTE\&hl=es\&sa=X\&ved=0ahUKEwjzt 96 7xpnkAhXGmq0KHUf6DooQ6AEIKTAA\#v=onepage\&q\&f=false

[15] Urdampilleta, A., López-Grueso, R., Martínez-Sanz, J. M. y MielgoAyuso, J. (2014). Parámetros bioquímicos básicos, hematológicos y hormonales para el control de la salud y el estado nutricional en los deportistas. Revista Española de Nutrición Humana y Dietética, 18(3), 155 - 171. doi: $10.14306 /$ renhyd
[16] Vega-Pérez, R., Ruiz-Hurtado, K. E., Macías-González, J., GarcíaPeña, M. D. y Torres-Bugarín, O. (2016). Impacto de la nutrición e hidratación en el deporte. El Residente, 11(2): 81-87. Recuperado de : www.medigraphic.com/elresidente 